\title{
Synchronization of Time-Delay Chaotic System in Presence of Noise
}

\author{
Min Lei*, Hai-peng Peng \\ Information Security Center, Beijing University of Posts and Telecommunications \\ National Engineering Laboratory for Disaster Backup and Recovery, Beijing University of Posts and \\ Telecommunications \\ Beijing, 100876, China \\ Chun-yu Yang \\ Institute of Electrical Engineering, Yanshan University \\ Qinhuangdao, 066004, China \\ Li-xiang Li \\ Information Security Center, Beijing University of Posts and Telecommunications \\ Beijing, 100876, China \\ Potsdam Institute for Climate Impact Research, \\ Potadam, 14473, Gemany \\ Received 5 November 2011 \\ Accepted 15 June 2012
}

\begin{abstract}
Chaotic synchronization, as a key technique of chaotic secure communication, has received much attention in recent years. This paper proposes a nonlinear synchronization scheme for the time-delay chaotic system in the presence of noise. In this scheme, an integrator is introduced to suppress the influence of channel noise in the synchronization process. The experimental results demonstrate the effectiveness and feasibility of the proposed scheme which is strongly robust against noises, especially the high-frequency noises.
\end{abstract}

Keywords: network security; chaotic synchronization; time-delay chaotic system

\section{Introduction}

Since Mackey and Glass firstly found chaos in timedelay system ${ }^{1}$, there has been increasing attentions to the researches on the time-delay chaotic systems ${ }^{2-4}$. In recent years, control and synchronization of chaotic systems have developed extensively ${ }^{5}$ due to their potential applications in many fields such as biological systems $^{6}$, chemical oscillators ${ }^{7}$, secure communication ${ }^{8}$, electronic systems ${ }^{9}$, and so on. Meanwhile, some distinguished synchronization methods are put forward, including Pecora-Carroll method ${ }^{10}$, observer-based approach $^{11}$, the adaptive method ${ }^{12}$ and network security.

Noise is ubiquitous in physical and natural systems. And the effect of the noise on dynamical systems has been a fundamental issue in nonlinear and statistical physics $^{13-17}$. It has been found that noise, under some conditions, can induce or enhance synchronization even in the absence of coupling ${ }^{15-17}$. However, noise is not beneficial to chaotic synchronization most of the time.

*Corresponding author: leimin@bupt.edu.cn 
Noise can also decrease or destroy the generalized synchronization, which depends on the details of the system such as the driving-driven configuration ${ }^{14}$.

In this paper, we study the influence of channel noise on the observer-based synchronization of timedelay chaotic systems and find that the synchronization error fails to converge at zero in the presence of noise. Therefore, in order to reduce the effects of channel noise, we introduce integrators to suppress noise and propose a novel synchronization scheme for the synchronization of time-delay chaotic systems. Under different frequencies of the noises, a lot of simulations are provided to verify the effectiveness and feasibility of the developed method. Simulation results show that the proposed method can successfully suppress the effects of high-frequency noise.

\section{Problem Formulation}

The model of transmitter based on the observer theory could be written as:

$$
\left\{\begin{array}{l}
\dot{x}(t)=A x(t)+B y_{1}(t)+F\left(y_{1}(t), x(t)\right)+G\left(y_{1}(t-\tau), x(t-\tau)\right) \\
y_{1}(t)=C x(t)
\end{array}\right\}
$$

where $x \in \mathfrak{R}^{n \times 1}$ is the state vector, $A \in \mathfrak{R}^{n \times n}$ and $B \in \mathfrak{R}^{n \times 1}$ are the parameter matrixes, $\tau$ is the time delay of system; $y_{1}$ is the scalar output, and $C \in \mathfrak{R}^{1 \times n}$ is the parameter matrix deciding the output signal $y_{1} ; F$ and $G$ are continuous nonlinear functions satisfying the following Lipschitz conditions:

$$
\begin{aligned}
& \left\|F\left(x_{1}\right)-F\left(x_{2}\right)\right\| \leq \rho\left\|x_{1}+x_{2}\right\|, \\
& \left\|G\left(x_{1}\right)-G\left(x_{2}\right)\right\| \leq \beta\left\|x_{1}+x_{2}\right\|,
\end{aligned}
$$

where $\rho$ and $\beta$ are both Lipschitz constants.

The state observer is modeled as the receiver, which is shown as follows ${ }^{8,11}$ :

$$
\begin{aligned}
& \dot{\hat{x}}=A \hat{x}(t)+B y_{1}(t)+F\left(y_{1}(t), \hat{x}(t)\right)+ \\
& G\left(y_{1}(t-\tau), \hat{x}(t-\tau)\right)+K\left(y_{1}-C \hat{x}(t)\right),
\end{aligned}
$$

where $\hat{x} \in \mathfrak{R}^{n \times 1}$ is the estimation of $x$, and $K$ is the observer gain.

The above observer-based method is an effective scheme in noiseless channels. However, experimental observations are always corrupted by channel noises. And we have to consider the effects of measurable additive noise on chaos synchronization. When there exists channel noise, we will obtain the signal as $\bar{y}=y_{1}+d$, where $y_{1}=C x$ and $d$ is the measured noise. In order to observe the influence of additive noise on the chaos synchronization, the simulation in Ref. [18] is undertaken here. We assume that there exist noises with zero mean, sample time 0.1 and amplitude 1 in the channel. The results of the above observer-based method (3) are depicted in Fig. 1. From Fig. 1, we know that the error curves fail to synchronize in presence of additive observational noises.

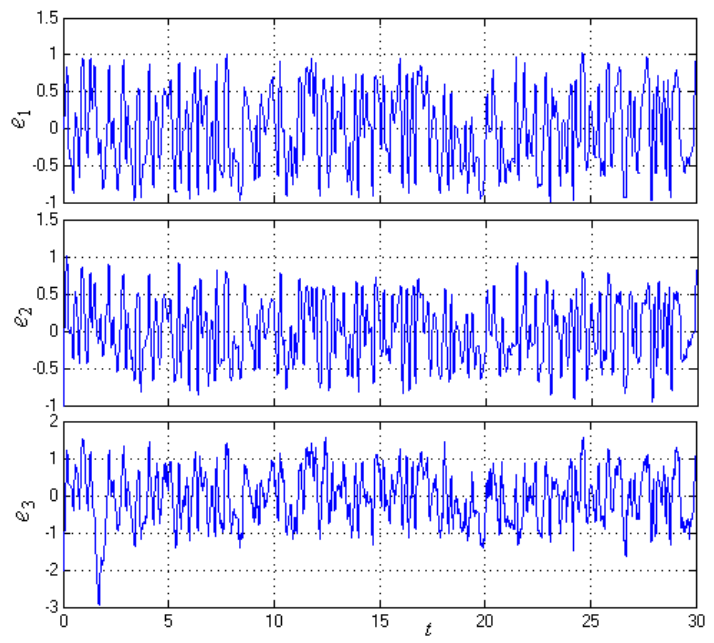

Fig. 1. (Color online) The error dynamics of synchronization between the drive system and the response system in presence of noise with zero mean, sample time 0:1 and amplitude 1 in the channel.

\section{Scheme in Presence of Channel Noises}

In order to improve the synchronization performance of time-delay chaotic system, we introduce the integrators, which are like low-pass filters, to suppress the noises $^{18,19}$. For chaotic system (1), we define a variable $\quad x_{0}=\int_{0}^{t} \bar{y}(\tau) d \tau \quad$, then we get $\dot{x}_{0}=\bar{y}=y_{1}+d=C x+d$. The improved system could be written as follows:

$$
\begin{aligned}
{\left[\begin{array}{c}
\dot{x} \\
\dot{x}_{0}
\end{array}\right]=} & {\left[\begin{array}{cc}
A & O \\
C & 0
\end{array}\right]\left[\begin{array}{l}
x \\
x_{0}
\end{array}\right]+\left[\begin{array}{cc}
B C & O \\
O & 0
\end{array}\right]+\left[\begin{array}{c}
F\left(y_{1}, x\right) \\
0
\end{array}\right]+} \\
& {\left[\begin{array}{c}
G\left(y_{1}(t-\tau) \cdot x(t-\tau)\right) \\
0
\end{array}\right]+\left[\begin{array}{l}
O \\
1
\end{array}\right] d }
\end{aligned}
$$

Let $Z=\left[\begin{array}{l}x \\ x_{0}\end{array}\right], \quad A_{1}=\left[\begin{array}{cc}A & 0 \\ C & 0\end{array}\right], \quad B_{1}=\left[\begin{array}{cc}B C & 0 \\ O & 0\end{array}\right]$, $F_{1}(z)=\left[\begin{array}{c}F\left(y_{1}(t), x(t)\right) \\ 0\end{array}\right], \quad G_{1}=\left[\begin{array}{c}G\left(y_{1}(t-\tau) \cdot x(t-\tau)\right) \\ 0\end{array}\right]$, 
$E=\left[\begin{array}{l}0 \\ 1\end{array}\right]$, then we have

$$
\left\{\begin{array}{c}
\dot{z}=\left(A_{1}+B_{1}\right) z+F_{1}(z)+G_{1}(z)+E d, \\
y_{0}=x_{0}=C_{1} z,
\end{array}\right.
$$

where $C_{1}=\left(\begin{array}{ll}O & 1\end{array}\right)$.

The response system is designed as

$\dot{\hat{z}}=\left(A_{1}+B_{1}\right) \hat{z}+F_{1}(\hat{z})+G_{1}\left(\hat{z}_{\tau}\right)+K\left(y_{0}-\hat{y}_{0}\right)$,

$\hat{y}_{0}=C_{1} \hat{z}, \hat{y}_{1}=C_{1} \hat{x}$,

where $F_{1}(\hat{z})=\left[\begin{array}{c}F\left(\hat{y}_{1}(t), \hat{x}(t)\right) \\ 0\end{array}\right]$,

$G_{1}\left(\hat{z}_{\tau}\right)=\left[\begin{array}{c}G\left(\hat{y}_{1}(t-\tau), \hat{x}(t-\tau)\right) \\ 0\end{array}\right]$.

Then we have

$\dot{e}=\left(A_{1}+B_{1}-K C_{1}\right) e+F_{1}(z)-F_{1}(\hat{z})+E d+G_{1}\left(z_{\tau}\right)-G_{1}\left(\hat{z}_{\tau}\right)$

where $e=z_{1}-\hat{z}$ and $\mathrm{K}$ is the observer gain.

Theorem 1. Assume $\left(A_{1}+B_{1}, C_{1}\right)$ is observable, and if the observer gain $\mathrm{K}$ satisfies

$$
\begin{aligned}
& A_{1}^{T} P+P A_{1}-P K C_{1}-C_{1}^{T} K^{T} P+B_{1}^{T} P+P B_{1}+\rho^{2} P^{2}+ \\
& \beta^{2} P^{2}+2 I+2 \delta P<0
\end{aligned}
$$

then the synchronization error could be small enough to guarantee the synchronization, where $\mathrm{P}$ is a positive definite and symmetric matrix, $I$ is the identity matrix, $\rho, \beta$ are the Lipschitz constants and $\delta$ is a positive constant. The synchronization error satisfies the following interval

$$
D_{0}=\left\{e:\|e\| \leq \frac{\gamma}{\delta} \cdot|d|+\mu\right\}
$$

where $\gamma=\frac{\left\|E^{T} P\right\|}{\lambda_{\text {min }}(P)}, \lambda_{\text {min }}(P)$ represents the smallest eigen value of matrix $\mathrm{P}$ and $\mu$ is a constant.

Proof: Let $V=e^{T} P e+\int_{t-\tau}^{t} e^{2}(s) d s$, then we have

$$
\begin{aligned}
\dot{V}= & \dot{e}^{T} P e+e^{T} P \dot{e}+e^{2}(t)-e^{2}(t-\tau) \\
= & {\left[\left(A_{1}+B_{1}-K C_{1}\right) e+F_{1}(z)-F_{1}(\widehat{z})+E d+G_{1}\left(z_{\tau}\right)\right.} \\
& \left.-G_{1}\left(\widehat{z}_{\tau}\right)\right]^{T} P e+e^{T} P\left[\left(A_{1}+B_{1}-K C_{1}\right) e+F_{1}(z)\right. \\
& \left.-F_{1}(\widehat{z})+E d+G_{1}\left(z_{\tau}\right)-G_{1}\left(\widehat{z}_{\tau}\right)\right]+e^{2}-e^{2}(t-\tau) \\
= & e^{T}\left(\left(\left(A_{1}+B_{1}-K C_{1}\right)^{T} P+P\left(A_{1}+B_{1}-K C_{1}\right)\right) e+\right. \\
& 2\left(F_{1}(z)-F_{1}(\widehat{z})\right)^{T} P e+2 E^{T} P e d+e^{2}-e^{2}(t-\tau)
\end{aligned}
$$

$$
+\left(G_{1}\left(z_{\tau}\right)-G_{1}\left(\widehat{z}_{\tau}\right)\right)^{T} P e+e^{T} P\left(G_{1}\left(z_{\tau}\right)-G_{1}\left(\widehat{z}_{\tau}\right)\right)
$$

Since $F_{1}(z)-F_{1}(\widehat{z})$ and $G_{1}\left(z_{\tau}\right)-G_{1}\left(\widehat{z}_{\tau}\right)$ satisfy the Lipschitz condition, so we have

$$
\begin{aligned}
\dot{V} \leq & e^{T}\left(\left(A_{1}+B_{1}-K C_{1}\right)^{T} P+P\left(A_{1}+B_{1}-K C_{1}\right) e+\right. \\
& 2 \rho\|e(t)\|\|P e\|+2\left\|E^{T} P\right\|\|e\||d|+e^{2}-e^{2}(t-\tau) \\
& +2 \beta\|e(t-\tau)\|\|P e\|
\end{aligned}
$$

Based on the equation $2 a b \leq a^{2}+b^{2}$, we have

$$
\begin{aligned}
\dot{V} \leq & e^{T}\left(\left(A_{1}+B_{1}-K C_{1}\right)^{T} P+P\left(A_{1}+B_{1}-K C_{1}\right)\right) e+ \\
& \|e\|^{2}+\rho^{2}\|P e\|^{2}+2\left\|E^{T} P\right\|\|e\||| d \mid+e^{2}(t) \\
& -e^{2}(t-\tau)+\|e(t-\tau)\|^{2}+\beta^{2}\|P e\| \\
= & e^{T}\left(A_{1}^{T} P+P A_{1}-P K C_{1}-C_{1}^{T} K^{T} P+B_{1}^{T} P\right. \\
& \left.+P B_{1}+\rho^{2} P^{2}+\beta^{2} P^{2}+2 I\right) e+2\left\|E^{T} P\right\|\|e\||d| \\
\leq & -2 \delta e^{T} P e+2\left\|E^{T} P\right\|\|e\||d| \\
\leq & -2 \delta \lambda_{\text {min }}(P)\|e\|^{2}+2\left\|E^{T} P\right\|\|e\||d| \\
= & -2 \delta \lambda_{\min }(P)\|e\|\left(\|e\|+\left\|E^{T} P\right\| /\left(\delta \lambda_{\text {min }}(P)\right) \cdot|d|\right) \\
= & -2 \delta \lambda_{\text {min }}(P)\|e\|\left(\|e\|-\frac{\gamma}{\delta} \cdot|d|\right)
\end{aligned}
$$

If $e$ does not belong to $D_{0}$, that is, $\|e\|>\frac{\gamma}{\delta} \cdot|d|+\mu$. Then for arbitrary $\|e\|>\frac{\gamma}{\delta} \cdot|d|+\mu$, we have $\dot{V} \leq-2 \mu \delta \lambda_{\text {min }}(P)\|e\|$. The proof is completed.

\section{Simulation research}

In this section, we utilize three representative examples to show that our scheme can be used to achieve synchronization in the time-delay chaotic systems in presence of noises.

\subsection{Example 1}

Firstly, we consider the time-delay system appearing in Ref. [8] which can be described by

$$
\begin{aligned}
& \dot{x}_{1}(t)=\alpha(1+\zeta) x_{2}-\alpha y_{1}+\alpha \delta \tanh \left(y_{1}-x_{2} \zeta\right), \\
& \dot{x}_{2}(t)=-(1+\zeta) x_{2}+x_{3}+y_{1}, \\
& \dot{x}_{3}(t)=\beta x_{2}-\gamma x_{3}+\varepsilon \sin \left(\sigma\left(y_{1}(t-\tau)-x_{2}(t-\tau) \zeta\right)\right), \\
& y_{1}(t)=x_{1}+\zeta x_{2},
\end{aligned}
$$


where $x_{1}, x_{2}$ and $x_{3}$ are the state vectors and $y_{1}$ is the scalar output. During the transmission, the output signal $y_{1}$ will be disturbed by noise and we assume that $d$ is the zero-mean noise in the channel. So we have $\bar{y}(t)=y_{1}(t)+d, y_{0}(t)=x_{0}(t)=\int_{0}^{t} \bar{y}(\tau) d \tau$. When $\alpha=9, \beta=14, \gamma=5, \tau=1, \delta=0.5, \varepsilon=10, \sigma=10^{4}$ and $\zeta=10^{-5}$, the system is chaotic and the response system could be designed as follows:

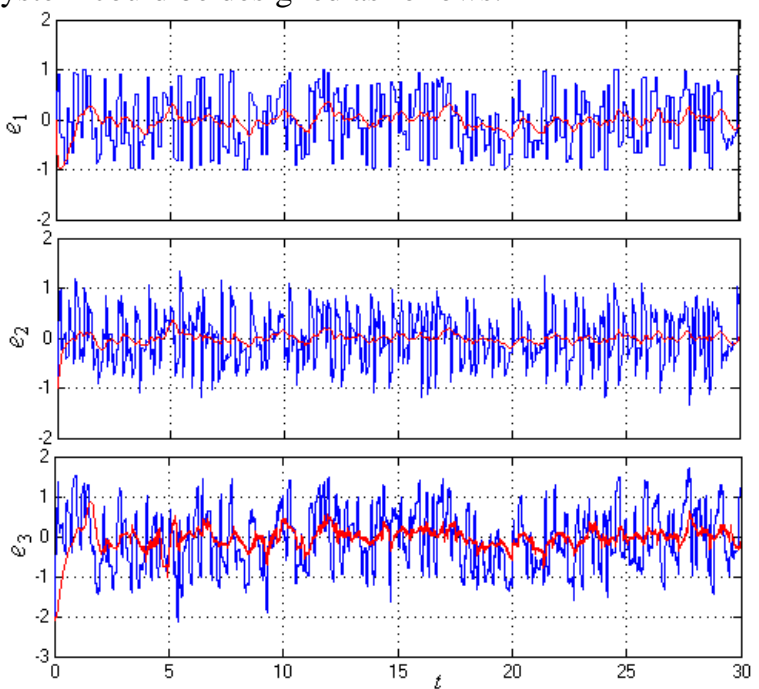

Fig. 2. (Color) The error dynamics of synchronization between the drive system and the response system for Example 1 in presence of noise with zero mean, sample time 0:1 and amplitude 1 in the channel. The blue curves represent the simulation results of observer-based method and the red curves show the synchronization results of the proposed scheme.

$$
\begin{aligned}
\dot{\bar{x}}_{1}(t)= & \alpha(1+\zeta) x_{2}-\alpha \hat{y}_{1}+\alpha \delta \tanh \left(\hat{y}_{1}-x_{2} \zeta\right)+k_{1}\left(y_{0}-\hat{y}_{0}\right) \\
\dot{\hat{x}}_{2}(t)= & -(1+\zeta) x_{2}+x_{3}+\hat{y}_{1}+k_{2}\left(y_{0}-\widehat{y}_{0}\right) \\
\dot{\bar{x}}_{3}(t)= & \beta x_{2}-\gamma x_{3}+\varepsilon \sin \left(\sigma\left(\hat{y}_{1}(t-\tau)-x_{2}(t-\tau) \zeta\right)\right)+ \\
& k_{3}\left(y_{0}-\hat{y}_{0}\right) \\
\dot{\bar{x}}_{0}(t)= & \widehat{x}_{1}+\zeta \widehat{x}_{2}+k_{4}\left(y_{0}-\hat{y}_{0}\right)
\end{aligned}
$$

where $k_{1}, k_{2}, k_{3}$ and $k_{4}$ are the observer gains and $\widehat{y}_{1}(t)=\widehat{x}_{1}+\zeta \widehat{x}_{2}$ is the output signal of the response system.

To compare the proposed scheme with the observerbased method in Ref. [18], we add noise and simulate the two schemes. The signals $e=x-\widehat{x}$ between the drive and response system is depicted in Fig. 2 and Fig. 3 , where $k_{1}=k_{2}=k_{3}=k_{4}=1000$. Fig. 2 and Fig. 3 show the experimental results in presence of noise with zero mean and sample time 0.1 and 0.01 , respectively.
From these figures, we can see that the synchronization results of the proposed scheme (red curves) are much better than those of the observer-based method (blue curves) in the aspect of stability.

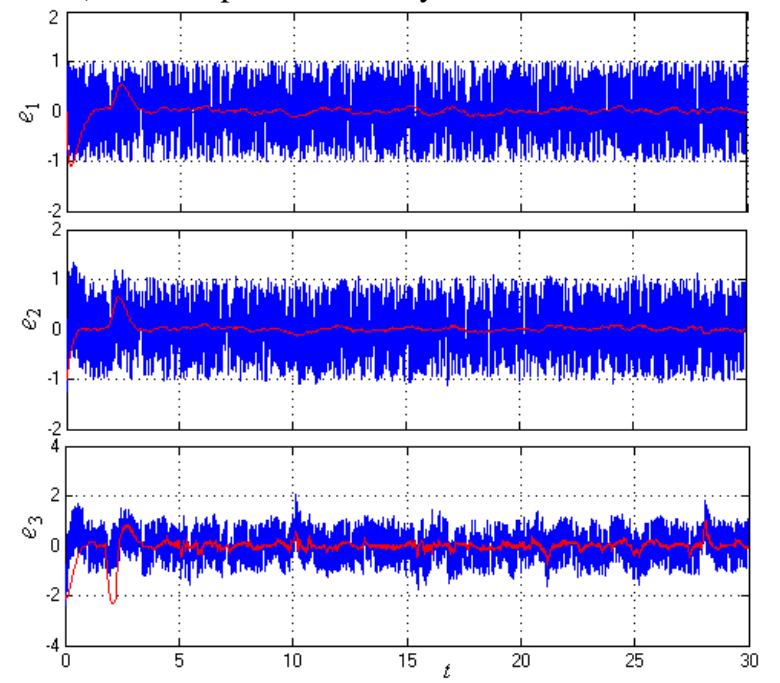

Fig. 3. (Color) The error dynamics of synchronization between the drive system and the response system for Example 1 in presence of noise with zero mean, sample time 0:01 and amplitude 1 in the channel. The blue lines represent the simulation results of observer-based method and the red lines show the synchronization results of the proposed scheme.

\subsection{Example 2}

Secondly, we adopt a polynomial approximation of the Mackey-Glass system, which can be described by [20]:

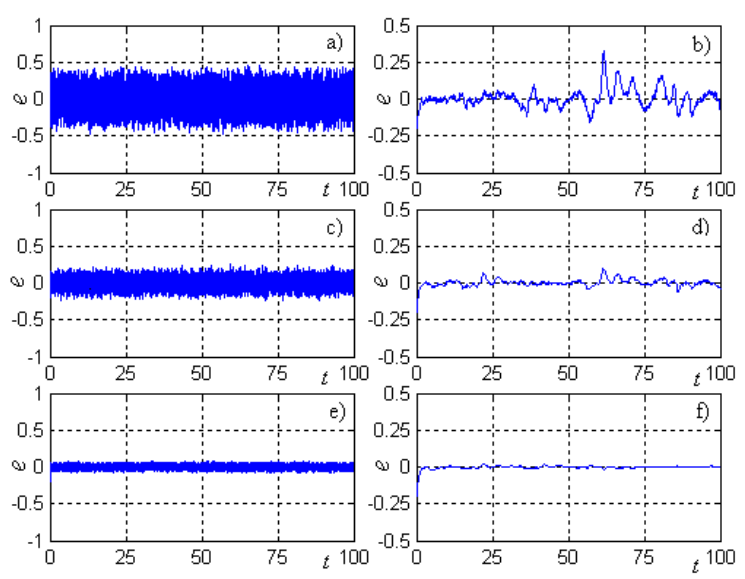

Fig. 4. (Color online) The synchronization error of Example 2 in presence of noise with zero mean and amplitude 0.5 , where 
(a), (c) and (e) show the error signals of the observer-based scheme and (b), (d) and (f) represent those of the proposed method. (a)-(b), (c)-(d) and (e)-(f) show the comparison curves between these two different methods when the sample times of noise are $0.01,0.001$ and 0.0001 , respectively.
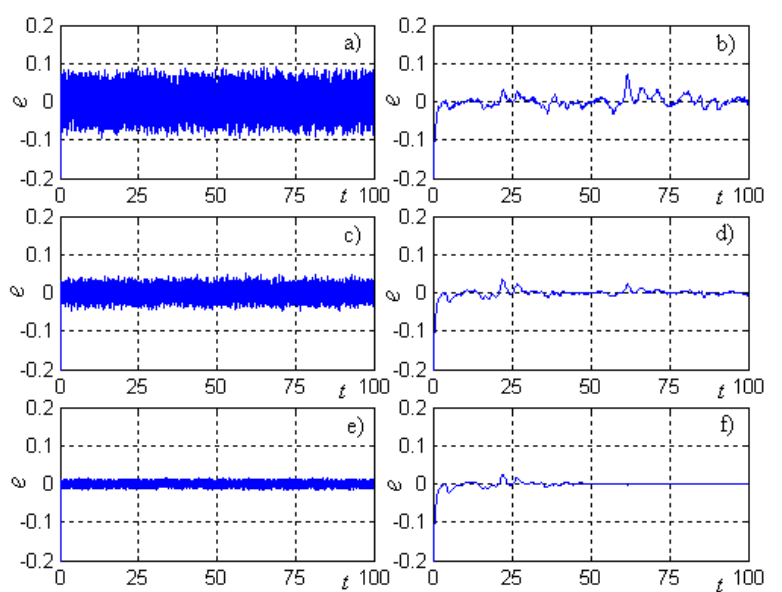

FIG. 5. (Color online) The synchronization error of Example 2 in presence of noise with zero mean and amplitude 0.1 , where (a), (c) and (e) show the error signals of the observer-based scheme and (b), (d) and (f) represent those of the proposed method. (a)-(b), (c)-(d) and (e)-(f) show the comparison curves between these two different methods when the sample times of noise are $0.01,0.001$ and 0.0001 , respectively.

$$
\begin{aligned}
& \dot{x}(t)=-a x+b\left(x(t-\tau)-x^{3}(t-\tau)\right), \\
& y_{1}(t)=x,
\end{aligned}
$$

where $y_{1}$ is the scalar output. When $\alpha=0.33, b=1.33$ and $\tau=4$, the system is chaotic. Consider $d$ as the noise added in the measurement output $y_{1}(t)$, then we have $\bar{y}(t)=y_{1}(t)+d$, $y_{0}(t)=x_{0}(t)=\int_{0}^{t} \bar{y}(\tau) d \tau$. The response system should be designed as:

$\dot{\bar{x}}(t)=-0.33 \widehat{x}+1.33\left(\widehat{x}(t-4)-\widehat{x}^{3}(t-4)\right)+k_{1}\left(y_{0}-\widehat{y}_{0}\right)$,

$\dot{\bar{x}}_{0}(t)=\widehat{x}+k_{2}\left(y_{0}-\hat{y}_{0}\right)$,

where $k_{1}$ and $k_{2}$ are the gains of observer.

The simulation results are shown in Fig. 4 and Fig. 5 when $k_{1}=k_{2}=100$ and the zero-mean noises have the amplitudes of 0.5 and 0.1 , respectively. In these two figures, sub-figure (a), (c) and (e) show the error curves of the observer-based scheme and sub-figure (b), (d) and (f) represent the error signals of our proposed method; sub-figure (a)-(b), (c)-(d) and (e)-(f) show the comparison results between two schemes when the sample times of noise is 0.01, 0.001 and 0.0001, respectively.

\subsection{Example 3}

Finally, we consider the system with the following mathematical form [20]:

$$
\begin{aligned}
& \xi \dot{x}=-x(t)+\frac{G}{1+\mu}\left(x(t-\tau)+U_{B}\right) \times[1+\mu \cos (\pi x(t-\tau)+ \\
& \left.\left.\quad U_{0}+U_{M}\right)\right], \\
& y_{1}(t)=x,
\end{aligned}
$$

where $y_{1}$ is the scalar output. When $U_{0}=2.0, U_{B}=1.0, U_{M}=-0.25, \mu=0.1 \quad$ and $\tau=0.1$ the system demonstrates the chaotic behavior. Adding zero-mean noise to output signal $y_{1}$, we have

$$
\bar{y}(t)=y_{1}(t)+d, y_{0}(t)=x_{0}(t)=\int_{0}^{t} \bar{y}(\tau) d \tau
$$

Accordingly, we design the response system as:

$$
\begin{aligned}
\xi \dot{\hat{x}}(t)= & -\widehat{x}(t)+(\widehat{x}(t-\tau)+1) \times[1+\cos (\pi \widehat{x}(t-\tau) \\
& -0.5)]+k_{1}\left(y_{0}-\widehat{y}_{0}\right), \\
\dot{\hat{x}}_{0}(t)= & \widehat{x}+k_{2}\left(y_{0}-\hat{y}_{0}\right),
\end{aligned}
$$

where $k_{1}$ and $k_{2}$ are the gains of observer.
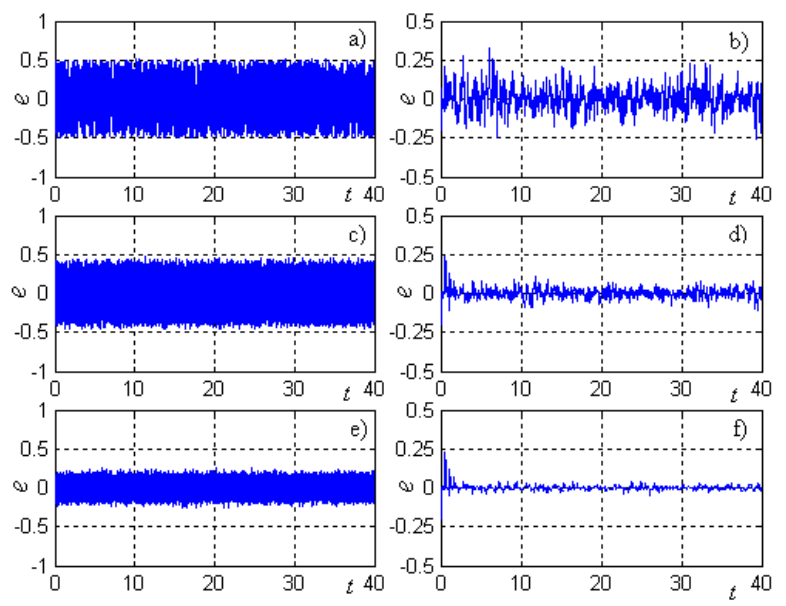

Fig. 6. (Color online) The synchronization error of Example 3 in presence of noise with zero mean and amplitude 0.5 , where (a), (c) and (e) show the error signals of the observer-based scheme and (b), (d) and (f) represent those of the proposed method. (a)-(b), (c)-(d) and (e)-(f) show the comparison curves between these two different methods when the sample times of noise are $0.01,0.001$ and 0.0001 , respectively. 
The simulation results are shown in Fig. 6 and Fig. 7 when $k_{1}=k_{2}=100$ and zero-mean noises have amplitudes of 0.5 and 0.1 , respectively. In these two figures, sub-figure (a), (c) and (e) show the synchronization errors of the observer-based scheme and sub-figure (b), (d) and (f) represent the error signals of our proposed method; sub-figure (a)-(b), (c)-(d) and (e)-(f) show the comparison results between these two schemes when the sample times of noise are $0.01,0.001$ and 0.0001 , respectively.

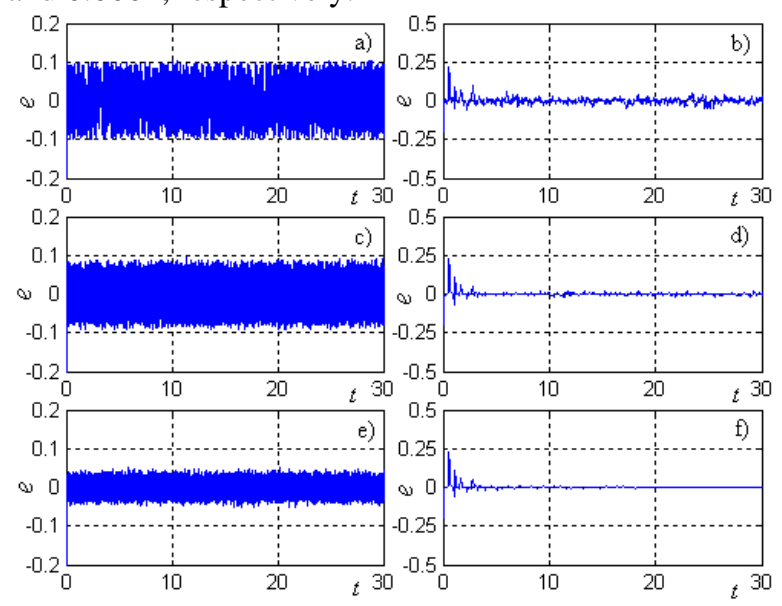

Fig. 7. (Color online) The synchronization error of Example 3 in the presence of noise with zero mean and amplitude 0.1, where (a), (c) and (e) show the error signals of the observerbased scheme and (b), (d) and (f) represent those of our method. (a)-(b), (c)-(d) and (e)-(f) show the comparison curves between these two different methods when the sample times of noise are $0.01,0.001$ and 0.0001 , respectively.

From Figs. 2-7, we can see that the synchronization curves of our scheme are smoother than those of observer-based scheme. Furthermore, from the comparison of the synchronization results, it can be inferred that the higher the frequency of $d(t)$ is, the better synchronization accuracy and the smoother error signal curves we can obtain. The integrator that functions with the low-pass filter ${ }^{18,19}$ can suppress the high-frequency noises. And that is the reason why the integrator is able to make the synchronization error smoother and decrease the impact of high-frequency noises.

In addition to the influence of noise frequency, the amplitude of noise is also a considerable factor affecting the results of the proposed scheme. Comparing Fig. 4 and Fig. 6 with Fig. 5 and Fig. 7, we can find that as the amplitude of noise increases, the results of both methods deteriorate and the errors oscillate more severely around 0 . In contrast to the proposed method, the observer-based scheme is relatively more sensitive to the increment of the amplitude of noise.

Note 1: The integrator and the low-pass filter can be designed by one-order RC circuit and the relationships between them can be consulted in Ref. [19]. Consequently, it is feasible to realize our method in practice.

Note 2: Noise is ubiquitous in physical and natural systems. Under noise conditions, the proposed integrator method can be popularized to the computational intelligence field and especially will be more effective when dealing with high-frequency noises.

\section{Conclusion}

In this paper, we consider the synchronization problem of time-delay chaotic systems in presence of noise. The synchronization performance of the observer-based approach in the presence of zero-mean noise is studied. We find that the synchronization errors could not converge to zero as time evolves. Therefore, In order to reduce the influence of noise on the synchronization results, the integrators are introduced and a novel scheme is proposed. Simulation results show that the proposed method can successfully suppress the effects of high-frequency noise. The proposed method can be further popularized to the fields of network security and computational intelligence.

\section{Acknowledgements}

This paper is supported by the National Natural Science Foundation of China under Grant No. 60973146, No. 61170269 and No. 61170272.

\section{References}

1. M. Mackey and L. Glass, Oscillation and chaos in physiological control system, in Science 197 (1977), pp. 287-289.

2. H. Lu and Z. He, Chaotic behavior in first-order autonomous continuous-time systems with delay, in IEEE Trans. Circ. Syst (1996), pp. 700-702.

3. M. Zigzag, M. Butkovski, A Englert, W Kinzel and I Kanter, Zero-lag synchronization of chaotic units with time-delayed couplings, in Europhys. Lett. (2009), 85, 60005. 
4. M. D. Shrimali, R. Sharan, A. Prasad and R. Ramaswamy, Delay-coupled discrete maps: Synhcronization, bistability, and quasiperiodicity, in Phys. Lett. (2010), pp. 2636-2639.

5. L. Li, H. Peng, Y. Yang and X. Wang, On the chaotic synchronization of Lorenz systems with time-varying lags, in Chaos Solitons Fractals (2009), pp. 783-794.

6. E. Mosekilde, B. Lading, S. Yanchuk and Y. Maistrenko. Bifurcation structure of a model of bursting pancreatic cells, in BioSystems (2001), pp. 3-13.

7. Y. N. Li, L. Chen, Z. S. Cai and X. Zhao. Experimental study of chaos synchronization in the BelousovZhabotinsky chemical system, in Chaos Solitons Fractals (2004), pp. 767-771.

8. E. Cherrier, M. Boutayeb and J. Ragot. Observers-based synchronization and input recovery for a class of nonlinear chaotic models, in IEEE Trans. Circ. Syst.-I 53 (2006), pp. 1977-1988.

9. N. F. Rulkov. Images of synchronized chaos: Experiments with circuits, in Chaos 6 (1996), pp. 262279.

10. L. M. Pecora and T. L. Carroll. Synchronization in chaotic systems, in Phys. Rev. Lett. (1990), pp. 821-824.

11. D. Ghosh. Nonlinear-observer-based synchronization scheme for multiparameter estimation, in Europhys. Lett. (2008), pp. 400-412.

12. S. Boccaletti, A. Farini and F. T. Arecchi. Adaptive synchronization of chaos for secure communication, in Phys. Rev. E (1997), pp. 4979-4981.

13. J. Ren, W. X. Wang, B. Li and Y. C. Lai. Noise bridges dynamical correlation and topology in coupled oscillator networks, in Phys. Rev. Lett. (2010), 104, 058701.

14. S. Guan, Y. C. Lai and C. H. Lai. Effect of noise on generalized chaotic synchronization, in Phys. Rev. E (2006), 73, 046210.

15. C. S. Zhou and J. Kurths. Noise-induced phase synchronization and synchronization transitions in chaotic oscillators, in Phys. Rev. Lett. (2002), 88, 230602.

16. W. Lin. Realization of synchronization in time-delayed systems with stochastic perturbation, in J. Phys. A (2008), 41, 235101.

17. L. Kocarev and Z. Tasev, Lyapunov exponents, noiseinduced synchronization, and Parrondo's paradox, in Phys. Rev. E (2002), 65, 046215.

18. G. P. Jiang, W. X. Zheng, W. K. S. Tang and et al., Integral-observer-based chaos synchronization, in IEEE Trans. Circ. Syst.-II (2006), pp. 110-114.

19. H. Peng, L. Li, Y. Yang and C. Wang, Parameter estimation of nonlinear dynamical systems based on integrator theory, in Chaos (2009). 19, 033130.

20. X. P. Guan, C. L. Chen, H. P. Peng and Z. P. Fan, Timedelayed feedback control of time-delay chaotic systems, in Int. J. Bifurcat Chaos (2003), pp. 193-205. 